
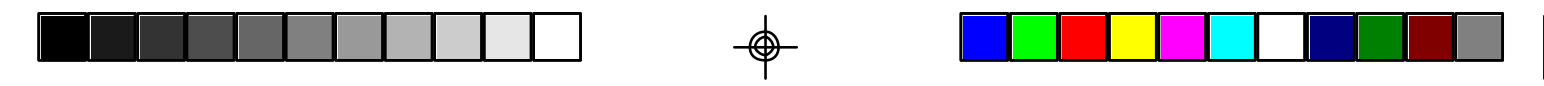

Cuadernos de Economía, Año 40, Nº 120, pp. 337-358 (Agosto 2003)

\title{
EL PRESTAMISTA DE ULTIMA INSTANCIA EN LA NUEVA INDUSTRIA BANCARIA
}

\author{
EDGARDO BARANDIARÁN*
}

\begin{abstract}
Since the mid-1970s, the banking industry has been changing rapidly, but our analysis of its regulation has been lagging. Leaving aside the sterile discussion about the intrinsic instability of the industry, today its regulation aims at preventing systemic risk. Little progress has been made, however, to agree on a definition of this risk. Exogenous risks associated with extreme events -such as macroeconomic shocks- have been separated from systemic risk, and now the idea of endogenous risk has been advanced. In some exceptional situations of no-coordinated interaction, endogenous risk may imply large losses to both banks and depositors. Although this idea does not support the assumption of rule-based commitments-even the loose commitments of constructive ambiguity-it supports the discretional intervention of a lender of last resort with government's approval.
\end{abstract}

\section{RESUMEN}

La transformación de la industria bancaria en las últimas tres décadas ha sido demasiado rápida y el análisis teórico y empírico de su regulación se ha quedado rezagado. Dejando de lado el debate estéril sobre la inestabilidad intrínseca de la industria, hoy la regulación tiene como objetivo inmediato la prevención del riesgo sistémico. Poco se ha avanzado, sin embargo, en lograr un consenso sobre su significado. Lentamente se ha progresado en separar los

Instituto de Economía, Pontificia Universidad Católica de Chile.

e-mail: ebarandi@faceapuc.cl

Agradezco los comentarios de Leonardo Hernández y Fernando Ossa.

Keywords: Lender of Last Resor, Central Banks, Commercial Banks, Financial Crises, International Monetary Fund.

JEL Classification: E58 y G28 
riesgos exógenos asociados a hechos extremos -en particular, a los shocks macroeconómicos-del concepto de riesgo sistémico. Ahora se propone la idea de riesgo endógeno, que en situaciones excepcionales de interacción no-coordinada podría implicar graves pérdidas patrimoniales para los bancos y los depositantes. Se argumenta que esta idea no apoya la pretensión de compromisos basados en reglas -aun los compromisos diluidos de la ambigüedad constructiva- pero justifica la intervención discrecional de un prestamista de última instancia, sujeta a la aprobación del poder político.

\section{INTRODUCCIÓN}

La profunda transformación de la industria bancaria en los últimos 25 años ha ido acompañada de cambios radicales en el sistema regulatorio de la industria. Cualesquiera hayan sido las relaciones entre los dos procesos, hoy la industria bancaria se caracteriza por una estructura concentrada que ofrece una gran variedad de servicios de pagos y servicios financieros. A su vez, el sistema regulatorio se caracteriza por un arsenal de instrumentos para mantener la estabilidad de la industria, en particular para prevenir el riesgo sistémico. Por cierto, la eficacia del sistema regulatorio continúa siendo motivo de intenso debate público y análisis académico. Mi propósito en esta nota es cuestionar la eficacia del instrumento más antiguo de ese arsenal: el gobierno como prestamista de última instancia de los bancos para resolver problemas de liquidez que amenazan la estabilidad de la industria.

La complejidad de la industria bancaria todavía plantea un gran desafío al análisis teórico y empírico. A pesar de la abundante investigación, el consenso de los académicos apenas alcanza a los enfoques metodológicos, y siempre reconociendo la necesidad de nuevos enfoques. La urgencia de consensos sobre los instrumentos apropiados de regulación de la industria no puede ocultar cuán lejos estamos de alcanzarlos; más aún, no debe ocultar que todavía no hay consenso sobre temas básicos. En esta nota, mi punto de partida es una revisión del estado actual de la investigación académica sobre estos temas básicos. Luego la revisión se extiende a la investigación más reciente sobre riesgo sistémico, cuya prevención hoy es motivo principal e inmediato de la regulación bancaria. En la sección siguiente, el análisis se centra en el gobierno como prestamista de última instancia de los bancos, en particular en las versiones más recientes que se han propuesto para asegurar su eficacia. Por último, a manera de conclusión, se argumenta a favor una posición precisa y constructiva sobre la regulación bancaria y se propone una intervención ex ante limitada a reglas precisas, complementada por intervenciones discrecionales ex post sujetas a la aprobación del poder político. En lugar de la intervención ex ante tradicional como prestamista de última instancia, las intervenciones expost incluyen la posibilidad de préstamos para hacer frente a problemas graves de liquidez de uno o más bancos. 


\section{La INDUSTRIA Bancaria: Existencia y Estabilidad}

El análisis teórico de la regulación de una industria cualquiera supone el conocimiento de los factores determinantes de las ofertas de las empresas. En ese análisis, algunas particularidades de la industria -entendida como el conjunto de empresas que ofrecen bienes o servicios sustitutivos a un grupo de usuarios potenciales- son decisivas para determinar la necesidad o la conveniencia de su regulación. La industria bancaria produce tres tipos de servicios -pagos, intermediación financiera y otros financieros- pero su sistema contable de pagos es su característica distintiva. ${ }^{1}$ Independientemente de cualquier otra razón para la regulación de los bancos, el sistema de pagos requiere de normas específicas para prevenir interrupciones y reponer los servicios en caso que se hayan interrumpido. Hoy la necesidad de estas normas es más notoria y urgente por la revolución de los medios electrónicos en los servicios bancarios de pagos, en particular en los pagos de alto valor. La realización del potencial de su sistema de pagos depende de esas normas.

Dejando de lado el sistema de pagos, el interés académico y político por la industria bancaria se centra en la intermediación financiera. El interés académico se concentra primero en explicar por qué existen intermediarios especializados en pedir prestado a un gran número de personas y en prestar a un gran número de empresas usando varios contratos de depósitos y préstamos. ${ }^{2}$ Las explicaciones son varias pero no mutuamente excluyentes y su importancia radica en que pueden implicar respuestas distintas a las preguntas sobre la estabilidad de la industria. En su reseña de la teoría, Gorton y Winton (2002) consideran a los bancos como mecanismos amortiguadores de shocks al consumo de las personas; productores de información sobre oportunidades de inversión; mecanismos para asumir compromisos sobre la inversión de los fondos de los depositantes; o monitores de las empresas deudoras en favor de los depositantes. ${ }^{3}$ El análisis teórico pretende mostrar que estas funciones requieren empresas especializadas y no pueden ser realizadas directamente entre las personas ahorristas y las empresas inversionistas, esto es, a través de los mercados de capital. Aunque estas explica-

$1 \quad$ Ver Barandiarán (2000).

2 En el análisis siguiente, cuando se hace referencia a depósitos se excluyen los depósitos a la vista porque son la base del sistema bancario de pagos. Por largo tiempo el análisis de la existencia de los bancos se concentró en la diferencia entre los bancos y otros intermediarios de fondos. En esta nota se supone que la diferencia está dada por los servicios de pagos. Este supuesto implica definir a los bancos como los intermediarios financieros que además prestan servicios de pagos. El análisis más reciente sobre la existencia de los bancos se concentra en explicar su papel de intermediario financiero $\mathrm{y}$, por lo tanto, la explicación es válida para otros intermediarios que no prestan servicios de pagos. La importancia relativa de los bancos en la intermediación financiera de los países varía mucho dependiendo de los efectos que la legislación ha tenido sobre el desarrollo de intermediarios no-bancarios.

3 Además, Gorton y Winton (2002) consideran a los bancos como proveedores de liquidez. Su revisión de los modelos teóricos que se centran en esta función de los bancos pone en evidencia que la provisión de liquidez es inherente al sistema bancario. 
ciones teóricas permiten entender aspectos de la actividad bancaria, la pretensión de mostrar que son exclusivas de la intermediación financiera no ha podido concretarse por las serias dificultades para la investigación empírica. ${ }^{4}$ La evidencia sobre las diferencias entre los bancos y los mercados de capital es fuerte, pero no prueba de que los bancos son suficientes para movilizar fondos desde los ahorristas a los inversionistas. 5

El creciente énfasis en los bancos como gestores de depósitos para ser invertidos en préstamos a empresas ha generado una literatura complementaria sobre la relación entre los bancos prestamistas y las empresas prestatarias. La literatura intenta explicar elementos centrales de esta compleja relación. La complejidad se expresa en la variedad de cláusulas incluidas en los contratos que podrían explicarse por la necesidad o conveniencia de dar incentivos para el cumplimiento de las obligaciones de las partes y por la responsabilidad de los bancos en cuanto monitores de sus deudores. Esta complejidad es extrema cuando sólo los bancos tienen acceso a información sobre las empresas prestatarias y las obligaciones de las partes se extienden más allá de las asumidas explícitamente en un momento y por un período determinado. Las relaciones complejas benefician a las empresas porque les facilita el acceso a fondos, pero al costo de quedar atrapadas por sus bancos acreedores y sujetas a la fortuna de los bancos. Aparentemente, la profundidad de las relaciones tiene consecuencias importantes para la organización y la estabilidad de la industria bancaria.

La idea de que la industria bancaria es intrínsecamente inestable ha servido para justificar la regulación de sus actividades. La inestabilidad se relaciona con un comportamiento procíclico de la cartera de préstamos y con una propensión de los depositantes a tener pánico de perder sus fondos. Tal comportamiento implica el agravamiento de las fluctuaciones en la actividad económica, y aunque el análisis teórico y la investigación empírica confirman la probabilidad de un efecto significativo, la regulación sólo puede pretender atenuar este efecto. ${ }^{6}$ El tema principal es la propensión a los pánicos porque implica la posibilidad de que hechos menores se transformen en crisis bancarias y económicas. Sobre esta posibilidad existe una abundante investigación histórica, pero poco ayuda a pre-

Más aún, la nueva industria bancaria se caracteriza por la venta de algunos de sus préstamos en los mercados secundarios a través de la emisión de otros títulos, lo que replantea el problema de la existencia de los bancos porque habría que explicar la decisión de las empresas inversionistas de pedir prestado a los bancos cuando podrían haberlo hecho directamente del mercado. Aunque esta nueva realidad ha favorecido las explicaciones que sostienen que los bancos son buenos monitores de las empresas deudoras en favor de los depositantes, su eficacia en el cumplimiento de esta función todavía no ha sido demostrada.

5 Esta evidencia está discutida en las secciones II.A y II.G de Gorton y Winton (2002).

$6 \quad$ Lo mismo puede decirse respecto de todas las actividades financieras, incluyendo las finanzas públicas. El carácter procíclico de estas actividades es consecuencia directa de su dependencia de las decisiones privadas y públicas de gastos. La intención de establecer mecanismos automáticos anticíclicos ha sido frustrada una y otra vez, tanto en las actividades privadas como en las finanzas públicas. 
cisar el problema y entender las condiciones para que los pánicos se transformen en corridas contra los bancos. ${ }^{7}$

El análisis teórico de los pánicos bancarios, indicativos de una industria intrínsecamente inestable, está muy limitado por los supuestos extremos que deben hacerse para obtener conclusiones precisas. Buena parte de este análisis está basado en el modelo de Diamond y Dybvig (1983), que supone que los bancos son mecanismos amortiguadores de shocks al consumo de las personas y que los depósitos son invertidos en activos irreversibles de duración indefinida. El supuesto adicional de que los depositantes están restringidos en el retiro de sus fondos por una regla secuencial del tipo "primero en llegar, primero en ser atendido" es crítico para que los depositantes corran cuando sus expectativas sobre la recuperación de sus fondos se vuelven negativas. En este tipo de modelo, algo extraño tiene que ocurrir (lo que en teoría se llama "manchas solares") para que se produzca un cambio repentino en las expectativas de los depositantes que los lleve a correr al banco. El pánico, o mejor dicho la corrida, es una solución del juego modelado por Diamond y Dybvig cuando se produce una "mancha solar". El modelo no identifica las "manchas solares" y entonces no es posible verificarlo empíricamente y evaluar la probabilidad de ocurrencia del fenómeno. Además, los bancos no están interrelacionados entre ellos, y por lo tanto, para que la corrida afecte a más de un banco simultáneamente, la "mancha solar" tendría que ser observada por los depositantes de otros bancos con el mismo efecto de cambiar repentinamente sus expectativas. Desde el punto de vista teórico, la crítica principal es que el supuesto sobre el retiro secuencial de depósitos es arbitrario, ${ }^{8}$ especialmente si la función de los bancos es la indicada por los autores. A pesar de los varios intentos de superar estas dificultades, poco se ha progresado porque pronto se tropieza con soluciones múltiples y la consecuente necesidad de introducir supuestos arbitrarios.

Los modelos teóricos que implican industrias bancarias inestables no han sido verificados directamente y sus conclusiones no son apoyadas por la investigación histórica. Aunque se argumenta que la historia de la industria bancaria de los Estados Unidos antes de la creación del Sistema de la Reserva Federal está plagada de corridas, los varios estudios de esta experiencia permiten concluir que la evidencia es contraria a las teorías que sostienen la inestabilidad intrínseca de

La falta de consenso sobre las definiciones apropiadas de pánico, corrida y crisis ha sido un tremendo impedimento para el análisis comparativo de la abundante investigación histórica. Según Gorton y Winton (2002), las definiciones estrictas de pánico y corrida contra un banco implican que han sido fenómenos excepcionales y sólo recurriendo a definiciones amplias que cubren casi todo tipo de crisis bancaria se puede concluir que su frecuencia no es menor. Sin embargo, la gran mayoría de las crisis bancarias se han debido a shocks macroeconómicos que afectaron simultáneamente a un número importante de bancos.

El hecho de que en circunstancias normales los retiros de fondos tengan lugar secuencialmente no implica que en circunstancias extraordinarias se aplicará una regla secuencial como la indicada en el texto. Sobre el modelo de Diamond y Dybvig, además de la revisión en Gordon y Winton (2002), ver los trabajos presentados en BIS (2002) que confirman la apreciación global presentada en el texto. 
los bancos. ${ }^{9}$ Los estudios de la experiencia de los Estados Unidos durante la Gran Depresión no apoyan estas teorías, aunque las conclusiones todavía son materia de fuerte discusión por las enormes dificultades de identificar la importancia de las intervenciones de la Reserva Federal y de los cambios en la actividad económica. Las experiencias de otros países tampoco apoyan esas teorías porque la incidencia de corridas ha sido poco importante, especialmente en comparación con la experiencia de los Estados Unidos. En los últimos 25 años, las crisis bancarias en varios países han motivado una nueva ola de estudios empíricos con poca o ninguna base teórica y no se puede inferir de ellos evidencia de que los sistemas bancarios nacionales han sido intrínsecamente inestables. Por el contrario, la evidencia muestra que estas crisis fueron provocadas por shocks macroeconómicos o por otros shocks fuera del control de los bancos, y por lo tanto su estudio puede a lo sumo ilustrar cómo el comportamiento de los bancos habría agravado las crisis, especialmente una vez iniciadas y luego que se hizo evidente que los bancos tendrían que realizar pérdidas.

En resumen, el análisis teórico y empírico de la industria bancaria ha permitido destacar que el desarrollo de la intermediación financiera no es un impedimento al desarrollo de los mercados de capital y viceversa. Ambos son formas alternativas de movilizar fondos para la inversión en empresas. Además, la evidencia empírica no apoya las teorías sobre la inestabilidad intrínseca de los bancos a pesar de la historia de corridas y crisis bancarias. En la sección siguiente supongo que la industria no es intrínsecamente inestable, aunque los riesgos de las actividades bancarias requieren una decisión política sobre si se justifica o no su regulación.

\section{La Industria B Ancaria: Regulación y Riesgo Sistémico}

Una interpretación muy libre de la historia de la regulación de la industria bancaria indica tres etapas. En un principio, desde mediados del siglo XIX hasta la Gran Depresión, el instrumento principal, sino único, de la regulación fue la intervención del gobierno como prestamista de última instancia cuando uno o más bancos enfrentaban problemas serios de liquidez. Luego, hasta mediados de la década de 1970, la regulación incluyó también varias restricciones a la entrada a la industria y especialmente a las actividades de intermediación financiera de los bancos. En esta etapa, el primer sistema formal de seguro de los depósitos se introdujo en los Estados Unidos y unos pocos países más. En la tercera etapa, primero se eliminaron muchas restricciones a la entrada a la industria y a las actividades de los bancos, pero luego el arsenal de instrumentos regulatorios aumentó con la extensión de los seguros de depósitos y especialmente con la adopción de las normas de Basilea en 1988. En este momento las nuevas normas de Basilea

Ver las secciones IV.A, B, C y G de Gorton y Winton (2002). Debe tenerse en cuenta que buena parte de la literatura teórica se ha desarrollado con el propósito explícito de explicar la experiencia de los Estados Unidos con anterioridad a 1914. 
están siendo discutidas y probablemente se adopten en 2004. El proceso acumulativo de instrumentos regulatorios implica que hoy el arsenal incluye la intervención del prestamista de última instancia (más otras intervenciones de los bancos centrales que no califican como última instancia $)^{10}$, el seguro de depósitos (o más precisamente garantías de los gobiernos a los depositantes), restricciones a la entrada a la industria (con variaciones importantes entre países que condicionan la competencia efectiva que tienen los bancos), restricciones a las actividades (incluyendo aquellas sobre las actividades de crédito), requerimientos mínimos de capital según las normas de Basilea, y procesos especiales para enfrentar problemas de solvencia.

La historia y la situación actual de la regulación bancaria se explica por una preocupación por la estabilidad de la industria, aunque en la segunda etapa (entre 1930 y 1975) muchas restricciones tuvieron por objetivo controlar la movilización de depósitos y la asignación de fondos prestables. La estabilidad de los bancos se relaciona con los riesgos de pérdidas patrimoniales por sus actividades. ${ }^{11}$ Una primera clasificación de estos riesgos implica distinguir entre riesgos exógenos y endógenos. Los riesgos exógenos son aquellos asociados a hechos no controlados por los bancos, mientras que los endógenos están asociados directamente al comportamiento normal de los bancos. Los primeros plantean problemas de seguridad a los bancos y los segundos pueden plantear problemas de seguridad a otros.

Los riesgos exógenos se asocian a hechos extremos y acciones criminales. Los hechos extremos son de naturaleza diversa y su importancia se puede ilustrar con las consecuencias del ataque terrorista a Nueva York y del shock a la macroeconomía chilena en 1982. En varios trabajos recientes se analizan los efectos del ataque terrorista sobre la economía de Nueva York. ${ }^{12}$ En su artículo, McAndrews y Potter (2002) muestran que la coordinación normal de los pagos colapsó por los daños extendidos a la infraestructura física del sistema de pagos y de las comunicaciones, pero la rápida respuesta del Banco de la Reserva Federal permitió reponer rápidamente el flujo normal de pagos. Igualmente, Fleming y Garbade (2002) explican que el ataque impidió a los vendedores de títulos del Tesoro de los Estados Unidos entregarlos oportunamente y en los días inmediatamente posteriores al 11 de septiembre de 2001 el incumplimiento de estas obligaciones aumentó sustancialmente. El Tesoro respondió con la emisión y venta de

Algunas de estas intervenciones están motivadas por problemas generalizados de liquidez y no con los problemas de liquidez de uno o varios bancos. Aunque se realicen a través de los bancos, estas operaciones son parte de la política monetaria y escapan a la regulación bancaria.

La teoría de la regulación del riesgo se refiere a acciones humanas que podrían causar daños a la integridad física de las personas y a sus patrimonios; ver Barandiarán (2003). Esta teoría está implícita en el análisis siguiente del riesgo sistémico y supone una distinción entre peligros asociados a acciones humanas y peligros derivados de hechos fortuitos que escapan al control de las personas y el gobierno.

12 Ver Economic Policy Review (publicación del Federal Reserve Bank of New York, Vol. 8, No. 2, November 2002). 
títulos con condiciones especiales para que los vendedores prefirieran pedirlos prestado en lugar de incumplir sus obligaciones. Más interesante, Bram y otros (2002) estiman que el costo económico del ataque fue 33-36 miles de millones de dólares hasta junio del 2002, pero los autores no hacen referencia a la posibilidad de que algunos bancos puedan haberse visto obligados a asumir parte de este costo. Por el contrario, el shock a la macroeconomía chilena en 1982 tuvo un costo económico alto, en la forma de una caída grave del producto interno por debajo de su nivel de pleno empleo aunque por un período corto, y afectó gravemente a los bancos, obligando a una intervención extraordinaria del gobierno para rescatar a las empresas deudoras de los bancos y de acreedores extranjeros, además de otras medidas que afectaron directamente a algunos bancos. ${ }^{13}$ En estos hechos extremos, los bancos no pudieron protegerse para disminuir las pérdidas y asegurarse para compartir las pérdidas con otros y, por lo tanto, la intervención de los gobiernos fue necesaria ex post para resolver rápido el problema de la distribución de las pérdidas y restablecer el funcionamiento normal de los bancos. ${ }^{14}$

Las acciones criminales también son fuente de riesgos exógenos. Las historias de Charles Ponzi y Willie Sutton en los Estados Unidos deben ser recontadas una y otra vez para no olvidar que los bancos están siempre amenazados por su apariencia de ser un blanco fácil para obtener un botín grande. Las estrategias de estos personajes fueron muy distintas, pero el resultado el mismo en términos del daño patrimonial causado. ${ }^{15}$ Ponzi, Sutton y muchos otros tuvieron intención criminal desde un principio, pero las acciones criminales también ocurren como parte de la reacción de los propietarios y gestores de los bancos a dificultades financieras debidas a hechos fortuitos. Frente a estas dificultades, la perspectiva de grandes pérdidas es un incentivo para que propietarios y gestores intenten evitar asumirlas personalmente y también aprovechar la situación para una ganancia neta.

Con respecto a los riesgos endógenos, el punto de partida es reconocer que la gestión de un banco, como cualquier otra acción humana, puede perjudicar a sus clientes, sus accionistas, otras personas relacionadas al banco y también a personas no relacionadas (por ejemplo, personas relacionadas a otros bancos).

14 Frente a los riesgos exógenos, los bancos pueden y deben autoprotegerse (tomar acciones para disminuir las pérdidas patrimoniales causadas por los hechos extremos), autoasegurarse (redistribuir en el tiempo las pérdidas patrimoniales) y/o asegurarse (compartir las pérdidas con otros pagando una prima). El análisis de estas opciones puede encontrarse en Ehrlich y Becker (1973). como el juego de Ponzi, que ilustra la situación explosiva que podría plantearse si un banco grande juega a mantener su liquidez ofreciendo tasas de interés a los depositantes mayores que las tasas de mercado. La historia de Willie Sutton es menos conocida y su famoso dicho "porque es donde la plata está" se conoce como la ley de Sutton o la ley de lo obvio. Sutton fue un ladrón de bancos cuya autobiografía se tituló That's where the money is. Recientemente su historia ha sido recordada por Della Paolera y Taylor (2002) en el análisis de la crisis argentina (curiosamente la figura de Ponzi fue rescatada en 1982 a propósito de otra crisis argentina). 
Independientemente de las expectativas frustradas sobre los beneficios de las relaciones con el banco, las deficiencias de la gestión bancaria que perjudican a personas relacionadas al banco en cuestión pueden ser motivo para un recurso judicial, pero sólo si se prueba culpabilidad el recurso puede tener éxito en lograr una compensación de pérdidas. Otras veces el perjuicio patrimonial es importante y los perjudicados pueden preferir la presión política al recurso judicial para lograr compensación de las pérdidas. Estas situaciones son importantes para los afectados y pueden precipitar una corrida contra el banco, y quizás "contagiar" a otro banco, pero difícilmente generen riesgo sistémico. Así, estas situaciones son riesgos endógenos pero no sistémicos.

El riesgo endógeno es sistémico cuando las interacciones de los bancos entre ellos y también entre los bancos y otras personas pueden causar perjuicios patrimoniales significativos. Esta posibilidad es resultado de una respuesta nocoordinada de los bancos y esas personas a un cambio exógeno en los determinantes fundamentales del comportamiento de la industria bancaria, y podría darse aunque este cambio fuera de poca cuantía. Aunque la respuesta de cada uno puede considerarse racional, las interacciones entre todos ellos generan una situación que puede llegar a ser crítica por la magnitud de las pérdidas. El mismo gobierno quizás intervenga, aunque no estuviera obligado o se hubiera comprometido a hacerlo. La posible intervención del gobierno tendría entonces que tomarse en cuenta para evaluar sus consecuencias sobre el "resultado" del juego, esto es, del conjunto de las interacciones.

Esa definición de riesgo sistémico se basa en Daníelsson y Shin (2002) que enfatizan cómo las personas y las empresas reaccionan a su entorno (esto es, a cambios en los determinantes fundamentales de su comportamiento) y cómo la reacción de cada persona o empresa afecta el entorno de los demás. ${ }^{16}$ Fenómenos

Aunque esta definición de riesgo sistémico se puede considerar un ejemplo de lo que De Bandt y Hartmann (2002) consideran situaciones "shocks limitados que se propagan y se transforman en un hecho sistémico, amplio y fuerte" o "shocks sistemáticos limitados que se transforman en un hecho sistémico, amplio y fuerte", su reseña de la extensa literatura sobre riesgo sistémico no hace referencia directa al tipo de riesgo endógeno que he denominado sistémico. Esta reseña de los varios conceptos de riesgo sistémico no concluye con una definición propia, aunque los autores terminan con una nota positiva: "Si hemos tenido éxito en convencer a los investigadores de cubrir algunas de las lagunas pendientes que hemos identificado en la literatura sobre riesgo sistémico, de manera que su trabajo pueda ser útil para explicar y prevenir crisis, entonces habremos logrado lo que esperábamos" (p. 289, mi traducción). Mi escepticismo sobre las lagunas que ellos habrían identificado es compartido por Goodhart e Illing (2002) en el capítulo introductorio donde se incluye el artículo de De Band y Hartmann; dicen Goodhart e Illing: "sus resultados, sin embargo, apuntan a una dificultad general para desarrollar test empíricos que puedan establecer una distinción clara entre los varios tipos de contagio [riesgo sistémico]" (p. 15, mi traducción; Goodhart e Illing dejan claro que la idea de contagio es esencial al concepto de riesgo sistémico). Más aún, el informe reciente sobre los trabajos presentados en la Tercera Conferencia Conjunta de Investigación de los bancos centrales deja establecida la gran variedad de situaciones que pretenden encuadrarse dentro del concepto de riesgo sistémico, y esto a pesar de que en el resumen del informe se da una definición según la cual riesgo sistémico es "el peligro de que los problemas de un banco puedan extenderse y, en situaciones extremas, tal 
incluidos en esta definición son posibles en otras actividades $;{ }^{17}$ pero en nuestro caso se pueden ilustrar con la experiencia chilena de 1982. Durante 1981, la situación macroeconómica fue cambiando: hasta el segundo trimestre, la economía se expandió a una tasa de crecimiento que había estado aumentando desde por lo menos un par de años atrás, pero ahí comenzó la desaceleración del crecimiento, que se haría más fuerte en los meses siguientes. A partir de julio de 1981, las explicaciones macroeconómicas dominaron el análisis y el debate: algunos sostuvieron que la política cambiaria no era consistente con otras políticas, otros sostuvimos que el aumento sostenido de las tasas de interés reflejaba simplemente la presión de una creciente demanda por fondos sobre una oferta que había dejado de crecer. A fines de 1981, los deudores de los bancos y de los acreedores extranjeros (incluyendo los grupos que controlaban varios bancos y empresas grandes) fueron optando por una estrategia de "salvataje", esto es, de mantener los activos adquiridos o en construcción con un mayor endeudamiento a tasas de interés crecientes. La estrategia sólo podía tener éxito si el gobierno intervenía o porque ya se había pasado el punto crítico para evitar una fuerte caída en los precios de los activos. A principios de 1982, la situación macroeconómica se agravó y pronto se entró en una recesión profunda, con un entorno externo muy desfavorable (las tasas de interés llegaron a niveles muy altos). Además, la primera intervención del gobierno (la devaluación de junio de 1982) forzó la insolvencia de muchas empresas, lo que luego requeriría otras intervenciones. En retrospectiva, aunque el shock macroeconómico causó la crisis, se puede argumentar que la severidad de la crisis económica fue consecuencia de la generalización de la estrategia de "salvataje". La respuesta de cada agente fue racional, aunque el juego podía conducir a un resultado no deseado por nadie. En aquel momento los intentos de modelar el fenómeno suponían que cada jugador no tomaba en cuenta la respuesta de los otros; en otras palabras, para cada jugador, las respuestas de los demás eran parte de un entorno que no podía influir. En todo caso, la severidad de la crisis fue determinada más por la fuerza del shock macroeconómico que por esas respuestas. ${ }^{18}$ Por supuesto, la evaluación ex ante de la magnitud del shock que la indus-

contagio perturbe el normal funcionamiento de todo el sistema financiero" (BIS, 2002, p. 1). La mayoría de los trabajos presentados a la Conferencia se relacionan con situaciones críticas que no pueden relacionarse con esta definición. De una u otra manera, la mayoría de los trabajos se refieren a shocks macroeconómicos, esto es, a lo que De Brand y Hartmann llaman shocks sistemáticos (afectan a buena parte del sistema económico y no sólo del sistema bancario) y que en el texto he incluido en los hechos extremos que generan riesgos exógenos al sistema bancario. en Londres, hecho ocurrido el 10 de junio de 2000. A la explicación dada por ellos sobre este hecho debe añadirse que se conocía sólo un caso anterior y que la probabilidad de que el Puente del Milenio colapsara por el balanceo agitado era casi cero. bastante mayor que el impacto reconocido en mis explicaciones de 1981-83. 
tria bancaria podría haber absorbido sin agravar su impacto macroeconómico es una tarea casi imposible.

Todavía es prematuro hablar de las consecuencias que la definición propuesta de riesgo sistémico puede tener para el análisis teórico de la regulación bancaria. Hoy el análisis continúa dominado por las dificultades para considerar simultáneamente dos o más instrumentos regulatorios y por el supuesto de que las decisiones de cada agente económico están determinadas por su propio entorno, sobre el cual no tiene influencia alguna. Esta situación está comenzando a cambiar porque las nuevas propuestas de Basilea sobre regulación bancaria están a su vez basadas en ese supuesto sobre las decisiones de los bancos y otros agentes económicos. Las críticas a las propuestas han sido más fuertes de lo esperado, quizás por el poco progreso en evaluar apropiadamente la experiencia con el acuerdo vigente (esto es, las normas que imponen un requerimiento mínimo de capital en base a los activos ponderados por riesgo y al riesgo de mercado medido por la metodología de value-at-risk). ${ }^{19}$

Según Daníelsson (2002a, 2002b), desde la perspectiva de la definición de riesgo sistémico antes propuesta, ${ }^{20}$ la eficacia del sistema actual y las nuevas propuestas de Basilea son cuestionables. El problema central es que si se toman en cuenta las interacciones estratégicas, las regulaciones pueden amplificar los efectos de un shock de menor cuantía sobre los precios y las cantidades, en particular su volatilidad puede ser muy fuerte y originar un círculo vicioso y terminar en una crisis bancaria. Su análisis, aunque todavía tentativo, apunta simplemente a la posibilidad de una crisis con y sin regulaciones. Como su análisis no considera otras intervenciones del gobierno en la industria bancaria, en particular

El primer acuerdo de 1988 introdujo la norma de un capital mínimo de $8 \%$ de los activos ponderados por riesgo. Las modificaciones de 1996 permitieron por primera vez el uso de modelos internos a los bancos usados para medir el riesgo de mercado. Las propuestas en discusión extienden los riesgos a considerarse en la determinación del capital mínimo para incluir riesgos de crédito y operaciones. Las propuestas también incluyen otros dos pilares para el sistema regulatorio: un proceso de revisión por los supervisores y la disciplina del mercado.

La aplicación del concepto de riesgo endógeno como sistémico al caso de la industria bancaria requiere modelar el fenómeno como un juego de información incompleta, en particular como un juego global. Muchos problemas económicos deberían modelarse como juegos de información incompleta, donde el pago de cada jugador depende de su propia acción, de las acciones de los demás jugadores y de fundamentos económicos desconocidos. Por el momento el análisis de estos juegos está limitado a situaciones relativamente simples. Los juegos globales son el resultado de una forma específica de simplificar los juegos de información incompleta. Los juegos globales suponen la identificación de entornos estratégicos con información incompleta, pero lo suficientemente ricos como para capturar el papel importante de las creencias de orden mayor en los fenómenos económicos y al mismo tiempo lo suficientemente simples como para poder analizarlos. En estos juegos se supone que la incertidumbre sobre las variables fundamentales del fenómeno en consideración se puede resumir en una única variable exógena y cada jugador observa una señal diferente de esta variable exógena, incluyendo un poco de ruido. Sobre juegos globales, ver la reseña de Morris y Shin (2000). 
las garantías de depósitos y los préstamos de última instancia, se podría argumentar que la combinación de todos estos instrumentos sí puede reducir casi a cero la probabilidad de una crisis como consecuencia del riesgo sistémico. Los otros instrumentos, sin embargo, también deben ser evaluados en términos de sus efectos sobre las interacciones estratégicas de los agentes económicos, y aunque la evidencia empírica sobre el riesgo moral de los seguros de depósitos y los préstamos de última instancia está lejos de ser concluyente, la posibilidad de que la combinación de instrumentos tenga un efecto menor o ninguno, o incluso perverso, sobre la probabilidad de una crisis bancaria debe ser considerada. Más aún, un análisis comparativo de las experiencias nacionales de los últimos 25 años no debe ignorar la posibilidad de una escalada regulatoria -para compensar el riesgo moral u otros efectos negativos de un instrumento, se introducen otros instrumentoscon consecuencias negativas sobre el acceso de un número importante de empresas a los servicios bancarios.

En resumen, hoy el análisis de la regulación bancaria está centrado en el riesgo sistémico, pero todavía el consenso sobre su significado es mínimo y la evidencia empírica sobre su importancia, discutible. Si se acepta que el concepto relevante se relaciona con las situaciones de riesgo endógeno discutidas antes, su importancia es mínima porque la gran mayoría de las crisis recientes han sido causadas por shocks fuertes que impactaron en varios sectores de la economía y por extensión en los bancos; la única duda es si todas las crisis se han debido a estos shocks y otros hechos extremos. ${ }^{21}$ Por otra parte, si el sistema regulatorio vigente se analiza tomando en cuenta las interacciones estratégicas que determinarían que el riesgo endógeno y sistémico fuera un peligro serio para la estabilidad de la industria, las conclusiones preliminares indican una seguridad ficticia. El sistema sigue siendo poco eficaz en prevenir este riesgo, aunque por suerte la exposición a este riesgo es mínima. La evaluación del sistema regulatorio debe también tomar en cuenta que el arsenal de instrumentos a disposición de los gobiernos se ha transformado, o se puede transformar, en una barrera para que los bancos tomen los riesgo de extender sus servicios a empresas "sin historia" bancaria.

Los trabajos recientes de Borio y otros investigadores del Bank of International Settlements están basados en que efectivamente la casi totalidad de las crisis bancarias se han originado en shocks macroeconómicos; ver, por ejemplo, Borio (2003) y Borio y Lowe (2002). El fracaso de los gobiernos en impedir que estos shocks y otros hechos extremos tuvieran consecuencias económicas graves, incluyendo crisis bancarias, es la motivación principal de muchas propuestas de políticas que están fuera del ámbito de la regulación bancaria y corresponden al ámbito de la macroeconomía, incluyendo las propuestas de Borio (2003). En todo caso, el punto importante es que si bien la industria bancaria puede ser un mecanismo significativo de la transmisión y extensión de los shocks macroeconómicos (de igual manera que todos los mercados financieros y la política fiscal son procíclicos), sólo la actuación discrecional ex post de los gobiernos puede ser eficaz en limitar las consecuencias de los shocks macroeconómicos. El problema principal es la persistencia de serias deficiencias en la capacidad de los gobiernos de actuar oportuna y apropiadamente, y esto a pesar del mejoramiento sustantivo en la disponibilidad de herramientas analíticas para predecir la ocurrencia de los shocks y la trayectoria de sus consecuencias. 

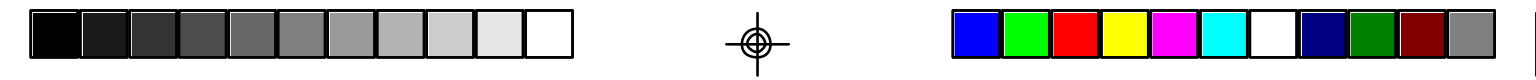

EL PRESTAMISTA DE ULTIMA INSTANCIA

\section{El Prestamista de Ultima Instancia}

Como se dijo antes, la regulación bancaria estuvo primero dominada por la intervención del gobierno como prestamista de última instancia. Hacia 1866, el Banco de Inglaterra ya había aceptado esta función y luego otros países hicieron lo mismo. Todavía se discute sobre la eficacia de estas intervenciones en las décadas anteriores a la Gran Depresión, y en particular su eficacia relativa en los países donde los bancos privados habían desarrollado mecanismos de cooperación para sus problemas de liquidez a través de sus cámaras de compensación de pagos. Luego, hasta mediados de la década de 1970, los bancos centrales ya establecidos en muchos países estaban preparados para actuar como prestamistas de última instancia, pero poco intervinieron porque las muchas restricciones a las actividades bancarias impusieron la estabilidad financiera al costo de reprimir su expansión. En los últimos 25 años, con la transformación de la industria, las crisis se sucedieron generando la percepción de un alto riesgo sistémico que ahora domina el análisis y el debate sobre la regulación bancaria. En esta nueva etapa, las intervenciones de los gobiernos a través de préstamos de última instancia y especialmente de programas especiales implicaron un alto costo fiscal. ${ }^{22}$ Así, la función de prestamista de última instancia ha vuelto a la agenda del análisis y el debate.

Las crisis bancarias de los últimos 25 años han sido motivo de mucha investigación empírica sobre sus causas y consecuencias. Como parte de esta investigación, la medición del costo fiscal y el costo económico de las crisis ha sido objeto de especial análisis, pero sólo las estimaciones del costo fiscal son confiables. Ningún estudio ha podido estimar razonablemente el costo económico de las crisis bancarias. Como lo reconocen Hoggart y Saporta (2001) en su estudio y revisión de la literatura: "El análisis teórico y empírico de las crisis sugieren que bajo ciertas condiciones las crisis bancarias pueden imponer grandes costos a la economía. Los estimadores del costo fiscal y el producto perdido parecen apoyar estas presunciones. Pero la cuantificación de estos costos, y la dirección de causalidad, están lejos de ser simples... [luego de referirse a los dos estudios más detallados de estos costos]. Sin embargo, en ambos estudios cabe la posibilidad de causalidad revertida, con grandes recesiones causando las crisis bancarias en lugar de las crisis causando recesiones más graves... En resumen, pareciera ser que a pesar de que, independientemente de si las crisis bancarias son causa o efecto de las recesiones, ellas agravan las pérdidas de producto..." (p. 160). Más aún, Hoggart y Saporta presentan sus estimadores de pérdida de producto de una manera totalmente neutra: "...over the past 25 years cumulative output losses during banking crises..." (resumen en p. 148); “...the broader welfare losses to the economy associated with a banking crisis..." (p. 152). Por lo tanto, afirmaciones como la siguiente no tienen base alguna en la investigación empírica: "las crisis bancarias no sólo han golpeado los presupuestos fiscales con gastos que han tenido que financiarse con mayores impuestos o reducciones en otros gastos, sino que también han tenido un alto costo económico en término de producto perdido" (Honohan y Klingebiel, 2001, p. 3; mi traducción). En resumen, la medición del costo económico de una crisis bancaria requiere haber identificado el impacto directo del shock macroeconómico que causó la crisis (la mayoría de las crisis fueron causadas por estos shocks), pero los modelos teóricos y la evidencia empírica no permiten esta identificación. 
Dado el contexto de la regulación bancaria hoy día, el papel del prestamista de última instancia no está claro. ${ }^{23}$ Hasta poco tiempo atrás, el grueso de la literatura sobre el prestamista de última instancia suponía que esta intervención era un sustituto total de las acciones privadas para enfrentar los problemas graves de liquidez de los bancos. Esta sustitución implicaba el "salvataje" (o bail out) de todos aquellos que podían perjudicarse si el problema de liquidez en realidad ocultaba un problema de solvencia. En otras palabras, se reconocía que la distinción teórica entre ambos problemas era imposible en la práctica. Como esta posición creaba un riesgo moral que podía ser serio, la posición extrema se fue abandonando en favor de un compromiso de los bancos centrales para intervenir de manera "ambigua pero constructiva". Luego veremos que la propuesta en sí misma es ambigua y no está claro que sea constructiva.

Otra alternativa a esa versión tradicional, originada en el contexto del debate sobre el papel del Fondo Monetario Internacional en el rescate de gobiernos insolventes (la exigencia de ajustes fiscales es la alternativa a la reestructuración o la liquidación de empresas privadas), ha planteado la idea del prestamista catalizador. ${ }^{24}$ La aplicación de esta idea a problemas de liquidez de los bancos implicaría la complementariedad entre los préstamos de última instancia y las acciones privadas. Para especular sobre esta aplicación y las condiciones bajo las cuales esta complementariedad podría darse, el modelo de Morris y Shin (2003) sobre el prestamista catalizador es una guía útil. ${ }^{25}$

Supongamos la existencia de tres partes interesadas en la industria bancaria y en particular en cómo se puede resolver un problema serio de liquidez de los bancos. Estas partes son los bancos, los depositantes y el gobierno. ${ }^{26}$ Cada una de las partes sabe que el resultado depende de sus propias acciones, de las acciones de las otras partes y de los cambios exógenos en los determinantes fundamen-

Goodhart e Illing (2002) presentan una colección de trabajos recientes sobre el prestamista de última instancia. En el artículo de Freixas y otros en esa colección se argumenta la necesidad de un prestamista como parte de la regulación bancaria, pero el concepto de riesgo sistémico que justificaría esta regulación no queda precisado.

Ver Cottarelli y Giannini (2002).

En el modelo de Morris y Shin, los jugadores son el gobierno deudor, sus acreedores de corto plazo y el Fondo Monetario Internacional. En mi adaptación, esos papeles corresponden a los bancos, los depositantes y el gobierno, respectivamente.

Podría suponerse que las empresas deudoras de los bancos también son una parte interesada y su comportamiento debiera tenerse en cuenta. Sin embargo, la importancia de las empresas deudoras en las crisis bancarias está generalmente relacionada al hecho de que la causa inmediata es un shock macroeconómico que afecta a un grupo importante de estas empresas. Frente a un shock macroeconómico, su impacto sobre los bancos vendrá tanto por el lado de los depositantes -la perspectiva de un menor ingreso probablemente los llevará a reducir sus depósitos- y también por el lado de las empresas deudoras que anticipan una menor capacidad de servir sus deudas. Por el contrario, en el texto el interés se centra en situaciones en que las acciones de las partes son respuestas a un shock de menor cuantía y por lo tanto no puede ser un shock macroeconómico. 

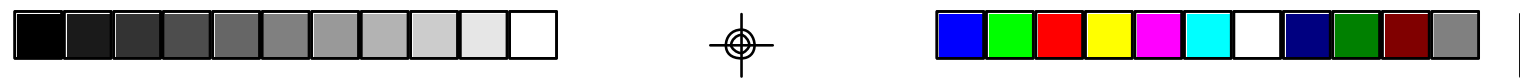

EL PRESTAMISTA DE ULTIMA INSTANCIA

tales de la solvencia de la industria bancaria. Comenzando con los bancos, cada banco tiene un monto $S$ de depósitos que vencen totalmente en cada período del análisis. Si los depósitos no son renovados, el banco puede pedir prestado al gobierno, o de lo contrario deberá entregar el control al gobierno. Para hacer frente al monto de depósitos no renovados $\sigma \mathrm{S}$, donde $\sigma$ es la proporción de los depósitos que no es renovada, el banco dispone de un flujo de caja $\theta$ que es la realización de una variable aleatoria con media $\phi+e$ y varianza $1 / \alpha$. La variable $\phi$ representa los determinantes fundamentales de la solvencia del banco y la variable $e$ representa el flujo adicional de caja que el banco puede obtener de un ajuste inmediato pero costoso en sus operaciones. ${ }^{27}$ El banco se supone que es solvente en el sentido de que el flujo de caja $\theta$ es mayor que el valor mínimo $\theta m$ necesario para mantener sus operaciones indefinidamente. El futuro del banco, sin embargo, está siempre en manos de sus depositantes en el sentido de que su flujo de caja $\theta$ tiene que ser mayor que $\theta m+\sigma S$. Si el banco no puede cubrir este monto, el gobierno tomará el control de sus operaciones.

Los depositantes renuevan sus depósitos, excepto cuando observan una señal sobre el flujo de caja $\theta$ y entonces reconsideran si lo harán o no. Dada la señal, su decisión dependerá del pago de una inversión alternativa y del pago esperado de renovar los depósitos sabiendo que el banco puede caer bajo el control del gobierno. Además, la señal puede preocupar al gobierno que podría intervenir como prestamista de última instancia del banco afectado bajo la condición de que haga algún ajuste en sus operaciones. Así, la interacción entre los depositantes y el gobierno es crítica para la resolución de un problema grave de liquidez. Si los depositantes creen que el préstamo del gobierno será decisivo para restablecer la certeza sobre la liquidez y la solvencia del banco, entonces estarían más predispuestos a renovar sus depósitos. Dada esta perspectiva, también el gobierno podría a su vez estar más predispuesto a intervenir y el banco a hacer algún ajuste en sus operaciones. En este caso, las acciones de las tres partes serían estratégicamente complementarias y generarían un círculo virtuoso. Por el contrario, la perspectiva de una intervención del gobierno puede no ser suficiente para persuadir a los depositantes a renovar sus depósitos y al banco a hacer el ajuste en sus operaciones, y entonces la intervención sería un sustituto estratégico de las reacciones de los depositantes y el banco. Los dos escenarios son posibles y tienen consecuencias muy distintas para evaluar el financiamiento catalizador.

Este ajuste es esencial para la intervención del gobierno. En la versión tradicional del prestamista de última instancia se exigía que los bancos entregaran garantías reales buenas (en el viejo dicho de Bagehot, había que prestar libremente a tasas altas de interés contra buenas garantías reales). Quizás tiempo atrás los mercados de crédito eran de difícil acceso aun para los bancos con buenas garantías reales; hoy esta exigencia ha perdido importancia porque cualquier banco con problemas de liquidez y buenas garantías reales puede acceder a los mercados de crédito y quizás ni siquiera necesite acceder a estos mercados para manejar sus problemas de liquidez. Hoy la exigencia importante es el ajuste de las operaciones del agente endeudado con problemas serios de liquidez. 
El análisis de esos dos escenarios debe hacerse en un contexto teórico que reconozca las interacciones estratégicas entre las tres partes. Morris y Shin (2003) recurren a la teoría de los juegos globales para simplificar su modelo y centrar el análisis en las decisiones de los acreedores de corto plazo (en nuestro caso, los depositantes) sobre si renovar o no sus títulos. En su simplificación, el Fondo Monetario Internacional (en nuestro caso, el gobierno) intenta intervenir sólo cuando la capacidad de pago del gobierno deudor se puede recuperar con un ajuste fiscal (en nuestro caso, cuando la incertidumbre sobre la solvencia de los bancos se puede recuperar con un ajuste en las operaciones). A su vez, el gobierno deudor (en nuestro caso, habría que suponer un único banco) tiene que decidir si hará o no el ajuste fiscal en anticipación de las reacciones de los acreedores de corto plazo y el Fondo Monetario Internacional. Las reglas impuestas por Morris y Shin para su juego global implican una solución que sólo para rangos bastante limitados de los parámetros críticos, el prestamista catalizador sería efectivo. ${ }^{28}$ Este resultado se explica porque los acreedores de corto plazo (los depositantes) 'exigen' un valor relativamente alto del flujo de caja $\theta$ para renovar sus títulos, y aunque la predisposición del FMI (el gobierno o banco central) a intervenir reduce este valor crítico del flujo de caja, el gobierno deudor (el banco) sólo estará dispuesto a hacer el ajuste fiscal (el ajuste de las operaciones) cuando el flujo de caja $\phi$ disminuya casi al nivel mínimo $\theta \mathrm{m}$ por un deterioro en los determinantes fundamentales.

Para Morris y Shin, la versión catalítica del prestamista de última instancia tiene un margen limitado de eficacia sin temor al riesgo moral de la versión tradicional. La aplicación anterior a los bancos no tiene en cuenta, sin embargo, que el gobierno tiene otras opciones. La opción de que el gobierno tome control del banco se mencionó, pero por la simplificación posterior no se tuvo en cuenta. Esta opción se puede implementar a través de la garantía de los depósitos por el gobierno y el traspaso del control al gobierno si la garantía se hace efectiva. La eficacia del instrumento dependerá primero de la percepción de los depositantes sobre el porcentaje de sus fondos que podrían recuperar en caso que la garantía se hiciera efectiva y, segundo, de la habilidad del gobierno para tomar oportunamente el control del banco y no dejar que el problema de liquidez degenere en un problema de solvencia. Si la percepción de los depositantes es fuerte tanto en cuanto al

Las reglas determinan que el juego proceda así. Primero, la "naturaleza" determina $\phi$ de una función de densidad $\mathrm{g}($.) y una vez determinado su valor es conocimiento común para todos. Segundo, el banco elige un nivel de ajuste de sus operaciones basado en su conocimiento de $\phi$ y el valor elegido de $e$ también es conocimiento común de todos. Tercero, el gobierno elige el monto $m$ que prestará al banco en base a los valores de $\theta$ y $e$, y el monto $m$ es anunciado públicamente, de manera que también es de conocimiento de todos. Cuarto, la "naturaleza" determina $\theta$ de una función normal con media $\phi+e$ y varianza $l / \alpha$, pero ninguna observa el valor verdadero de $\theta$. Quinto, cada depositante observa la realización de una señal $x=\theta+\epsilon$ donde $\epsilon$ es normal y donde los "ruidos" de dos pares cualquiera de depositantes son independientes; la información de cada depositante consiste entonces en las variables $\theta, e, m$ y su señal privada $x$ y en base a esta información decide si renueva o no su depósito. 
porcentaje de recuperación de fondos como a la certeza de la recuperación, los depositantes muy probablemente renovarán sus depósitos y en este sentido la garantía es un mecanismo mejor que los préstamos de última instancia. Si el gobierno interviene de manera oportuna y decisiva, el riesgo moral de la garantía de los depósitos se minimizará y no será una desventaja fuerte respecto al prestamista de última instancia. ${ }^{29}$ En relación a la versión catalítica, la garantía de los depósitos parece más eficaz.

La segunda alternativa al prestamista de última instancia es el relajamiento temporario de algunas restricciones a los bancos. Los efectos de este relajamiento son difíciles de precisar si no se identifican las restricciones. Como sustituto del prestamista de última instancia, su eficacia depende de cómo afecte el flujo adicional de caja asociado a un ajuste en las operaciones y de su costo para el banco. Tiempo atrás el ejemplo más sencillo de este tipo de acción habría sido la rebaja de los encajes legales sobre los depósitos, pero hoy la suspensión del requerimiento mínimo de capital sería una opción. En ambos casos la diferencia principal con los préstamos de última instancia es que evitan el riesgo moral de estos últimos, aunque no totalmente. Como en los préstamos, el gobierno tomaría la decisión en función de las circunstancias en que se plantee el problema de liquidez, pero sin arriesgar fondos públicos. Si pasado el tiempo de excepción el banco no pudiera cumplir con las restricciones en el plazo otorgado, el gobierno podría entonces proceder a tomar el control del banco con un margen mayor de maniobra por no haber arriesgado todavía fondos públicos. El costo para el banco depende principalmente de la duración de la excepción en relación al desarrollo esperado de su problema de liquidez.

La evaluación comparativa de los varios instrumentos de regulación bancaria en el contexto de interacción estratégica antes mencionado es una tarea pendiente. Esta comparación se complica además por las dificultades para precisar ex ante los detalles sobre cómo se aplicaría cada instrumento. En el análisis teórico se simplifica para poder mostrar la existencia de soluciones y en particular de soluciones únicas. Las simplificaciones, sin embargo, tienden a ignorar los problemas de información que enfrenta el gobierno para su aplicación razonable. Un error común en algunos análisis teóricos es suponer que los problemas de información de los agentes privados se solucionan a través de las exigencias de los órganos públicos, pero la poca capacidad de estos órganos para verificar y genera el problema de liquidez, pero en la medida en que la intervención es discrecional, el Banco Central siempre tiene un período de negociación en que puede reducir ese riesgo. En el caso de la garantía de depósitos, suponiendo que no tiene efectos negativos de largo plazo en la forma de riesgo moral, una vez que el banco enfrenta el problema de liquidez queda en manos del banco actuar de manera oportunista, y si el gobierno no reacciona pronto un resultado posible es que se transforme rápidamente en un problema de insolvencia de gran magnitud. La experiencia de las crisis bancarias (y también de las dificultades financieras de las empresas) muestra que el momento crítico para las pérdidas finales es cuando el banco debe decidir cómo reaccionar a las señales de que enfrenta dificultades financieras. 
analizar la información recibida debe ser tomada en cuenta e inclina la balanza en favor de aquellos instrumentos menos exigentes en información.

El análisis anterior cuestionó la eficacia de compromisos asumidos por el gobierno para intervenir en caso que los bancos enfrenten problemas graves de liquidez. Aunque el gobierno no haya asumido compromiso alguno, su intervención ex post es siempre una posibilidad. La constitución y las leyes condicionan esta intervención pero no la impiden. Sin conocer los detalles de todas las democracias constitucionales, el margen para esta intervención es lo suficientemente amplio para que los gobiernos puedan hacerlo si obtienen la aprobación exigida por la constitución y las leyes. En el caso específico del prestamista de última instancia, si ex ante no hay compromiso alguno, ex post el gobierno puede decidir hacerlo. De hecho, el alto costo fiscal de las crisis bancarias de los últimos 25 años es resultado de decisiones ex post. ${ }^{30}$ Los mecanismos preventivos implicaban un costo fiscal muy inferior pero fracasaron, y los gobiernos intervinieron una vez que las crisis estaban avanzadas. Las dificultades para diseñar mecanismos preventivos eficaces permiten suponer que las intervenciones ex post seguirán siendo decisivas en la resolución de las crisis bancarias, y en un sentido amplio de las dificultades financieras de los bancos en el contexto de las políticas de ajuste a shocks macroeconómicos. El factor decisivo de la intervenciónex post es la capacidad para hacerlo de una manera oportuna y apropiada.

Las propuestas de un prestamista de última instancia a la vez ambiguo y constructivo pretenden introducir un grado de incertidumbre en los compromisos del prestamista. El temor al riesgo moral derivado de compromisos precisos ha llevado a suponer que si estos compromisos se diluyen a través de condicionarlos a procesos de revisión y aprobación, entonces los bancos actuarán más prudentemente. Las propuestas mismas son ambiguas y evitan pronunciarse sobre si implican o no algún compromiso de manera que pueda hablarse de una intervención ex ante. ${ }^{31}$ En el mejor de los casos, estas propuestas implican delegar en un órgano administrativo (el Banco Central o el supervisor de bancos) la responsabilidad de intervenir ex post para resolver los problemas de liquidez. Por sus características las propuestas específicas sobre cómo introducir incertidumbre no pueden ser analizadas seriamente. El análisis sólo puede evaluar los incentivos que las propuestas incluyen para que los responsables de las decisiones cumplan con sión; ver Kashyap (2002).

31 Un ejemplo claro de propuesta ambigua es la recomendación de un informe del Grupo de los 10 que dice: “...cualquier compromiso a un curso específico de acción en apoyo de una institución financiera debe ser evitado por los gobiernos, que deben retener discreción sobre sí, cuándo y cómo intervenir. Además, cuando se tomen esas decisiones, es importante analizar si existe una amenaza sistémica y, si existe, analizar qué opciones puede haber para hacer frente a los efectos del contagio sistémico de manera de limitar el impacto adverso sobre la disciplina de los mercados" (Bank of International Settlements, 1997). Si bien se ataca al compromiso a un curso específico, no se precisa si la discreción mencionada supone el ejercicio de un poder expresamente delegado en los bancos centrales para hacerlo. 
los objetivos de la delegación de poder. Esta evaluación no se ha hecho a pesar de la abundancia de modelos teóricos sobre la delegación de poder, pero esos incentivos serían el único elemento constructivo que las propuestas podrían incluir.

En resumen, a pesar de sus limitaciones, el análisis anterior permite llegar al problema principal en el diseño de mecanismos apropiados para la intervención del prestamista de última instancia. Una vez que se reconocen las consecuencias de asumir compromisos precisos y las limitaciones de los mecanismos de intervención ex ante, el problema es si el legislador debe o no delegar poder en un órgano administrativo -quizás independiente del presidente- para que decida ex post la intervención. Las mismas dificultades que hacen inconveniente asumir compromisos también incomodan la definición del mandato del órgano administrativo. El mandato tendría que ser amplio, pero esto aumentaría el costo de monitorear la gestión del órgano. Estas consideraciones apuntan, por lo menos tentativamente, a que el órgano debería tener responsabilidad sólo por preparar las propuestas de intervención (esto es, para tomar la iniciativa), quedando la aprobación en manos del presidente y en situaciones extremas del legislador.

\section{Conclusión}

La transformación de la industria bancaria ha sido demasiado rápida y el análisis teórico y empírico se ha quedado rezagado. Hacia 1970, el estancamiento y la estabilidad de los 25 años anteriores habían facilitado un progreso significativo en el análisis de una industria relativamente simple y reprimida por una variedad de restricciones legales. Hoy el análisis debe distinguir por lo menos tres funciones principales de los bancos: los servicios de pagos que en un futuro desplazarán casi totalmente a las monedas; los servicios de intermediación financiera; y los otros servicios financieros. En las secciones anteriores me he referido exclusivamente a los servicios de intermediación financiera porque el análisis hoy está centrado en ellos. El debate sobre la regulación bancaria no puede, sin embargo, ignorar las otras dos funciones. En particular, no puede ignorar la necesidad de normas mínimas para asegurar el funcionamiento eficaz de los servicios bancarios de pagos. Las propuestas de regulación basadas en la idea de narrow banking pretenden separar los servicios de pagos de los servicios de intermediación financiera, pero esta separación difícilmente pueda implementarse. La integración de las dos funciones -en realidad, de las tres funciones- es una de las varias tareas pendientes del análisis de la regulación bancaria.

En relación a la intermediación financiera, su regulación supone ante todo una definición clara de su objetivo. Dejando de lado el debate académico sobre la presunta inestabilidad intrínseca de la industria bancaria, hoy la regulación tiene como objetivo inmediato la prevención del riesgo sistémico. Otros riesgos o peligros pueden requerir normas adicionales para reforzar y facilitar el cumplimiento de las normas generales que regulan las actividades empresariales -en particular los riesgos exógenos asociados a acciones criminales y los riesgos endógenos asociados a errores en la gestión bancaria. Por el contrario, el riesgo sistémico 
sería algo propio de la industria y extendido a buena parte de ella. A pesar de las innumerables referencias al riesgo sistémico, poco se ha avanzado en lograr un consenso sobre su significado. Lentamente se ha progresado en separar los riesgos exógenos asociados a hechos extremos -en particular a los shocks macroeconómicos-del concepto de riesgo sistémico. Esta separación se dificulta porque la investigación empírica sólo ha puesto en evidencia que las crisis bancarias de los últimos 25 años han sido causadas por hechos extremos. En la búsqueda de un significado preciso para el concepto de riesgo sistémico ahora se propone la idea de riesgo endógeno, que en determinados contextos de interacción nocoordinada podría implicar graves pérdidas patrimoniales para los bancos y los depositantes. ${ }^{32}$

El poco progreso en definir el objetivo de la regulación bancaria se refleja en el análisis del arsenal de instrumentos de que hoy disponen los gobiernos. Las nuevas propuestas de Basilea han servido para intensificar las críticas al sistema vigente, pero el análisis teórico y empírico todavía está lejos de ofrecer un apoyo adecuado para el diseño de intervenciones ex ante dirigidas a prevenir el riesgo sistémico. Esta situación quizás justifique la reticencia a abandonar instrumentos aparentemente ineficaces, pero que no se ha probado sean contraproducentes. La desregulación de la industria ha significado la eliminación de restricciones cuyo propósito sólo era afectar la captación y la asignación de fondos, pero no otros instrumentos. Así, el actual sistema regulatorio refleja una acumulación de instrumentos para asegurar la estabilidad de la industria, aunque poco se conozca sobre la eficacia de cada uno de ellos y del conjunto. La idea de que nuevos instrumentos han sido necesarios para contrarrestar los efectos negativos de instrumentos existentes es a menudo mencionada en el debate, pero el análisis no permite conclusiones firmes sobre esta necesidad y mucho menos sobre la eficacia de los nuevos instrumentos.

En ese contexto, la distinción entre intervenciones ex ante y ex post para prevenir el riesgo sistémico es importante. Las primeras suponen compromisos precisos -no la ambigüedad propuesta para combatir el riesgo moral de los instrumentos viejos- y, los segundos, la autoridad discrecional para actuar oportuna y eficazmente. El análisis tendría entonces que orientarse a depurar el arsenal de intervenciones ex ante y a desarrollar las herramientas que permitan las intervenciones ex post. El rechazo absoluto a las intervenciones ex post porque suponen una discrecionalidad más o menos amplia no tiene fundamento cuando las inter-

En sus introducciones a la Tercera Conferencia Conjunta sobre Investigación de los Bancos Centrales, tanto A. Crockett (Gerente General, Bank of International Settlements) y Y. Yamaguchi (Vice-Gobernador del Banco de Japón) hicieron referencia al riesgo endógeno, enfatizando aspectos de la definición usada en el texto; ver BIS (2002), especialmente páginas 14 y 18. Sólo unos pocos trabajos presentados en la Conferencia aplicaron esta definición, sin embargo. Cabe notar que en relación a los desarrollos teóricos recientes, Yamaguchi saca una conclusión muy distinta a la presentada en el texto; según Yamaguchi estos desarrollos apoyan su convicción de que la globalización de los mercados financieros y los sistemas bancarios implican que las intervenciones ex ante son preferibles a las intervenciones ex post. 

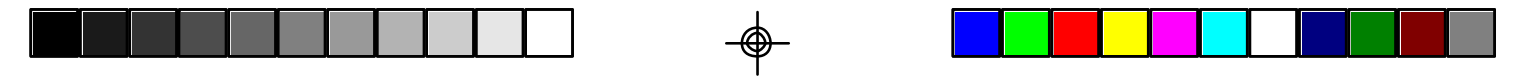

EL PRESTAMISTA DE ULTIMA INSTANCIA

venciones ex ante no son eficaces, más aún cuando los peligros que se quiere prevenir son excepcionales, tan excepcionales como los estados de emergencia reconocidos por las constituciones políticas. En particular, toda intervención de emergencia que requiera montos importantes de recursos fiscales debe plantearse como intervención discrecional y ex post, sujeta a la aprobación del poder político, sea el presidente o el legislador (presidente más congreso). Por cierto, desde la perspectiva de esta conclusión, el análisis del prestamista de última instancia debería prestar especial atención a la organización y el ejercicio de la autoridad para intervenir ex post de una manera oportuna y eficaz.

\section{REFERENCIAS}

Bank of International Settlements (1997), G 10 Report on Financial Stability in Emerging Market Economies, Basilea.

Bank of International Settlements (2002), Risk Measurement and Systemic Risk: Proceedings of the Third Joint Central Bank Research Conference, BIS: Committee on the Global Financial System, October 2002.

Barandiarán, E. (1988), "La Gran Recesión de 1982", en F. G. Morandé y K. SchmidtHebbel (editores), Del Auge a la Crisis de 1982, ILADES.

Barandiarán, E. (2000), "Chile Después del Peso: Viviendo con el Dólar", Cuadernos de Economía, Vol. 37 No. 110, 241-267.

Barandiarán, E. (2003), "The Theory of Risk Regulation and the Precautionary Principle", borrador.

Barandiarán E. y L. Hernández (1999), "Origins and Resolution of a Banking Crisis: Chile 1982-1986", Banco Central de Chile WP 057.

Borio, C. (2003), "Towards a macroprudential framework for financial supervision and regulation?", BIS Working Papers No. 128, February.

Borio, C. y P. Lowe (2002), "Assessing the risk of banking crises", BIS Quarterly Review, December 2002, 43-54.

Bram, J., J. Orr y C. Rapaport (2002), "Measuring the Effects of the September 11 Attack on New York", Federal Reserve Bank of New York: Economic Policy Review, November 2002, Vol. 8 No. 2, 5-20.

Cottarelli, C. y C. Giannini (2002), "Bedfellows, Hostages, or Perfect Strangers? Global Capital Markets and the Catalytic Effect of IMF Crisis Lending", IMF, WP No. 02/193, November 2002.

Daníelsson, J. (2002a), “On the Feasibility of Risk Based Regulation”, London School of Economics, draft paper, September 2002.

Daníelsson, J. (2002b), "The emperor has no clothes: Limits to risk modelling”, Journal of Banking \& Finance, Vol. 26, 1273-1296.

Daníelsson J. y H. S. Shin (2002), “Endogenous Risk”, London School of Economics, draft paper, September 21, 2002.

Daníelsson J. y J-P. Zigrand (2002), "What Happens When You Regulate Risk? Evidence from a Simple Equilibrium Model", London School of Economics, draft paper, October 2002. 
De Bandt, O. y P. Hartmann (2002), "Systemic Risk in Banking: A Survey”, en C. Goodhart y G. Illing, Financial Crises, Contagion, and the Lender of Last Resort, Oxford University Press.

Della Paolera G. y A. Taylor (2002), "Gaucho Banking Redux”, draft paper.

Diamond, D. y P. Dybvig (1983) "Bank Runs, Deposit Insurance and Liquidity”, Journal of Political Economy, Vol. 91, 401-419.

Ehrlich, I. y G. Becker (1973) "Market Insurance, Self-Insurance and Self-Protection”, Journal of Political Economy, Vol., 623-648.

Fleming, M. J. y K. D. Garbade (2002), "When the Back Office Moved to the Front Burner: Settlement Fails in the Treasury Market after 9/11", Federal Reserve Bank of New York: Economic Policy Review, Vol. 8 No. 2, 35-58.

Goodhart, C. y G. Illing (2002), Financial Crises, Contagion, and the Lender of Last Resort, Oxford University Press.

Gorton G. y A. Winton (2002), "Financial Intermediation", The Wharton Financial Institutions Center, WP 02-28 (forthcoming in G. Constantinides, M. Harris and R. Stulz, Handbook of the Economics of Finance, North Holland).

Hoggarth, G. y V. Saporta (2001), "Costs of banking system instability: some empirical evidence", Bank of England Financial Stability Review, June, 148-165.

Honohan, P. y D. Klingebiel (2001) “Controlling Fiscal Costs of Banking Crises”, World Bank, draft paper.

Kashyap, A. K. (2002), “Sorting Out Japan's Financial Crisis”, NBER Working Paper 9384.

McAndrews, J. J. y S. M. Potter (2002), "Liquidity Effects of the Events of September 11, 2001”, Federal Reserve Bank of New York: Economic Policy Review, Vol. 8 No. 2, 59-79.

Morris, S. y H. S. Shin (2000), “Global Games: Theory and Applications”, draft paper, July.

Morris, S. y H. S. Shin (2003), “Catalytic Finance: When Does It Work?”, draft paper, January 10 . 